\title{
Linearized Riccati Equation as a Tool for Nonlinear Optics of Weakly Excited Two-Level Systems
}

\author{
R. PARZYŃSKI* AND T. KOPYCIUK \\ Faculty of Physics, A. Mickiewicz University, Uniwersytetu Poznańskiego 2, 61-614 Poznań, Poland \\ (Received October 27, 2019; in final form March 2, 2020)

\begin{abstract}
Linearized version of the Riccati-type differential equation for the ratio of population amplitudes in a laserdriven two-level system is used to calculate analytically the induced electric dipole moment. The formula found for the dipole moment is valid for weak excitations by smooth-shape pulses of arbitrary off-resonant frequencies, i.e., those producing neither exact one-photon nor odd multiphoton resonances. For a given pulse shape, the formula allows to express the Fourier components of the induced dipole moment as functions of both laser frequency and intensity and to find the field-dependent refractive index of the system versus laser frequency.
\end{abstract}

DOI: 10.12693/APhysPolA.137.1080

PACS/topics: two-level system, induced electric dipole moment, harmonic generation, Riccati-type equation, nonlinear optics

\section{Introduction}

The model of two discrete levels is known [1] to serve as the most popular paradigm for different phenomena from the scope of light-matter interaction. Despite its simplicity, the model gives deep understanding of both resonant and off-resonant processes because it can be solved analytically in an approximate way even if no rotating wave approximation is made. In the last 25 years, this twolevel model has been extensively used in, e.g. theoretical investigation of the role played by bound-bound transitions in generation of high harmonics of light from the matter exposed to linearly polarized laser field [2-21]. Generation of harmonics of a given light beam is a key phenomenon in nonlinear optics because this process is a source of new radiation of often much higher frequency than that of the primary beam. Such new radiation is required for experimental spectroscopy, for example. In the investigation mentioned, both the regimes of weak excitations [5, 9, 19] and strong excitations [3, 5, 8, 17, 19] were considered. For both regimes the main aim of the papers cited above was to calculate the induced electric dipole moment in order to analyse the photon-emission spectrum of the system. To name a few, we point to some approaches used in the calculations, e.g. the optical Bloch equations $[2,7,8,12,17,21]$, the appropriately transformed equations for the level population amplitudes $[3,9,11]$, the Mathieu-type differential equation for the amplitudes [5], the Floquet-Green formalism [18] and the Riccati-type equation for the ratio of the population amplitudes [19, 20].

The present paper is a significant extension of Sect. 4.1 of our previous paper [19], where weakly excited two-level

*corresponding author; e-mail: parzynsk@amu.edu.pl model was solved analytically by applying the Riccatitype differential equation for population amplitudes, but under two restrictive approximations, i.e., the approximation of square temporal profile of the laser pulse and the approximation of low laser frequency. The first approximation idealized the real laser pulse, completely neglecting its finite turn-on and turn-off times, while the other assumed the laser frequency to be much lower than the transition frequency (the so-called multiphoton excitation regime). Due to these restrictions the applicability of our previous solution was strongly limited.

Now, we take into consideration both smooth temporal profile of the laser pulse and arbitrary frequency of light. Mathematically, it is much more challenging task. Nevertheless, we shall find an analytical solution covering much broader scope of applicability than previously. As we are interested in the regime of weak excitations too, we shall solve the so-called linearized form of the exact Riccati-type differential equation and then use this solution to derive an analytical formula for the induced electric dipole moment. Particular forms of the formula for the dipole moment will be obtained corresponding to different laser frequency and laser strength limits. Also, amplitudes of harmonics generated and field-dependent refractive index of the system will be discussed as functions of laser frequency.

\section{Riccati equation and its linearization}

For a two-level system in a laser field, the Riccati-type equation is that for the ratio $R=C_{2} / C_{1}$. Here, $C_{1}$ and $C_{2}$ are the time-dependent population amplitudes of the two opposite-parity field-free states, i.e., the lower state 1 (initially occupied) and the upper state 2 (initially empty), respectively. This differential equation is of the form $[19,22]$ :

$$
\text { i } \dot{R}=Q(t) R^{2}-Q^{*}(t),
$$


and results from the pair of equations for the statepopulation amplitudes [23]:

$$
\begin{aligned}
& \mathrm{i} \dot{C}_{1}=-Q(t) C_{2}, \\
& \text { i } \dot{C}_{2}=-Q^{*}(t) C_{1},
\end{aligned}
$$

where the dots over $R$ and $C_{j}$ stand for the time derivatives. In the electric dipole approximation for the interaction Hamiltonian but without employing the rotating wave approximation,

$$
Q(t)=\Omega_{R} f(t) \cos \left(\omega_{0} t\right) \mathrm{e}^{-\mathrm{i} \omega_{21} t}
$$

with $\omega_{21}=\omega_{2}-\omega_{1}$ being the transition frequency, the laser-field frequency $\omega_{0}$, the smooth envelope of the laser pulse $0 \leq f(t) \leq 1$, and the standard Rabi frequency $\Omega_{R}=\boldsymbol{\mu}_{21} \cdot \varepsilon_{0} / \hbar$ expressed by the dipole transition matrix element $\boldsymbol{\mu}_{21}=\langle 2|$ er $|1\rangle$ and the amplitude $\varepsilon_{0}$ of the electric field of the linearly polarized beam.

The variable $R$ from (1) gives complete information about the population evolutions, $\left|C_{1}\right|^{2}$ and $\left|C_{2}\right|^{2}$, and the induced electric dipole moment $\boldsymbol{d}(t)=\langle\Psi(t)|\operatorname{er}| \Psi(t)\rangle$, where $\Psi(t)=C_{1} \exp \left(-\mathrm{i} \omega_{1} t\right)|\rangle+C_{2} \exp \left(-\mathrm{i} \omega_{2} t\right)|\rangle$ is the state vector of the laser-driven two-level system. In terms of $R$, the state populations and the induced dipole moment are expressed as

$$
\begin{aligned}
& \left|C_{1}\right|^{2}=\frac{1}{1+|R|^{2}}, \\
& \left|C_{2}\right|^{2}=\frac{|R|^{2}}{1+|R|^{2}}, \\
& \boldsymbol{d}(t)=2 \boldsymbol{\mu}_{12} \operatorname{Re}\left(C_{1} C_{2}^{*} \mathrm{e}^{\mathrm{i} \omega_{21} t}\right)=2 \boldsymbol{\mu}_{12} \frac{\operatorname{Re}\left(R \mathrm{e}^{-\mathrm{i} \omega_{21} t}\right)}{1+|R|^{2}}
\end{aligned}
$$

where we made use of the conservation law for the total population probability, $\left|C_{1}\right|^{2}+\left|C_{2}\right|^{2}=1$.

Throughout this paper we focus on weak excitations, $\left|C_{2}\right|^{2} \ll 1$, meaning $|R|^{2} \ll 1$. In this case, the term quadratic in $R$ in (1) is much smaller than the other term on the right-hand side and we can use the approximate procedure proposed in [19]. In short, we initially drop out this quadratic term and start from the zero-order solution to (1):

$$
R_{0}(t)=\mathrm{i} \int_{t_{0}}^{t} Q^{*}\left(t^{\prime}\right) \mathrm{d} t^{\prime} .
$$

Then, the $R^{2}$ term is included by the substitution $R=R_{0}+R_{1}$ with the restriction that $\left|R_{1}\right| \ll\left|R_{0}\right|$. This two-part $R$, when substituted to (1), gives a different Riccati-type equation but for $R_{1}$ now. However, rejecting the smallest $R_{1}^{2}$ term in the equation for $R_{1}$, we reduce this nonlinear equation to the linear first order differential equation

$$
\text { i } \dot{R}_{1}=2 Q R_{0} R_{1}+Q R_{0}^{2} \text {. }
$$

As distinct from Eq. (1), Eq. (9) has the exact solution

$$
R_{1}(t)=-\frac{1}{2} \int_{t_{0}}^{t} R_{0}\left(t^{\prime}\right) \frac{\mathrm{d} Z\left(t, t^{\prime}\right)}{\mathrm{d} t^{\prime}} \mathrm{d} t^{\prime},
$$

where

$$
Z\left(t, t^{\prime}\right)=\exp \left(2 \int_{t^{\prime}}^{t} \dot{R}_{0}^{*}\left(t^{\prime \prime}\right) R_{0}\left(t^{\prime \prime}\right) \mathrm{d} t^{\prime \prime}\right) .
$$

The above solution for $R_{1}$ can be expressed in a slightly different form using the relations $\dot{R}_{0}=\mathrm{i} Q^{*}$ and $\dot{R}_{0}^{*}=-\mathrm{i} Q$. Equations (8) and (10) give a formal solution $R=R_{0}+R_{1}$ to (1) in the case of weak excitations. The procedure leading to such a solution is called the linearization of the starting Eq. (1).

In [22], Rostovtsev et al. have presented an alternative linearization procedure to the above one. In their approach, the $R^{2}$ term in (1) was replaced by $R^{2}=\left(R-R_{0}\right)^{2}+2 R_{0} R-R_{0}^{2} \approx 2 R R_{0}-R_{0}^{2}$. However, by putting $R=R_{0}+R_{1}$ one converts such approximated Eq. (1) for $R$ into our Eq. (9) for $R_{1}$. It means that the two linearization procedures [19, 22] of Eq. (1) are equivalent. Rostovtsev et al. have shown numerically that this linearization procedure gives accurate results for the excitation probability.

\section{Calculation of $R_{1}(t)$}

The integrals over time in (8), (10) and (11) can be calculated analytically for an arbitrary smooth-shape function $f(t)$, but in an approximate way. To this end, we make use of the two properties of $f(t)$, i.e., it is zero at the initial time $t_{0}$ and is, together with its powers, the slowest function as compared to the other timedependent functions in integrands when no exact oddphoton resonances occur in the system. Assuming the lack of such resonances, we perform the integrals by parts and neglect the emerging integrals including the time derivatives of both $f$ and higher powers of $f$.

Beyond one-photon resonance, i.e., for $\omega_{0} \neq \omega_{21}$, Eq. (8) for $R_{0}$ gives along this line

$$
R_{0}(t)=\frac{x}{y^{2}-1} f(t) \mathrm{e}^{\mathrm{i} \omega_{21} t}\left[y \cos \left(\omega_{0} t\right)-\mathrm{i} \sin \left(\omega_{0} t\right)\right],
$$

where $x=\Omega_{R} / \omega_{0}$ and $y=\omega_{21} / \omega_{0}$ are dimensionless field-strength and field-frequency parameters, respectively. According to the applied linearization procedure, it has to be $\left|R_{0}\right|^{2} \ll 1$ entailing the limitation

$$
x^{2} \ll f_{1}(y)=2 \frac{\left(y^{2}-1\right)^{2}}{y^{2}+1},
$$

where we have replaced $\cos ^{2}\left(\omega_{0} t\right)$ and $\sin ^{2}\left(\omega_{0} t\right)$ by their time average values and $f^{2}(t)$ by its maximum value. In the limiting case of low laser frequencies $(y \gg 1$, multiphoton excitation) Eq. (13) leads to $(x / y)^{2} \ll 2$, while for high laser frequencies $(y \ll 1)$ to $x^{2} \ll 2$. For other laser frequencies, fixed in $y$, the strength parameter $x$ fulfilling Eq. (13) can be estimated from Fig. 1 (solid line).

Using (12) for $R_{0}$ and $\dot{R}_{0}^{*}=-\mathrm{i} Q$, we find that the exponent in $Z\left(t, t^{\prime}\right)$ of (11) is 


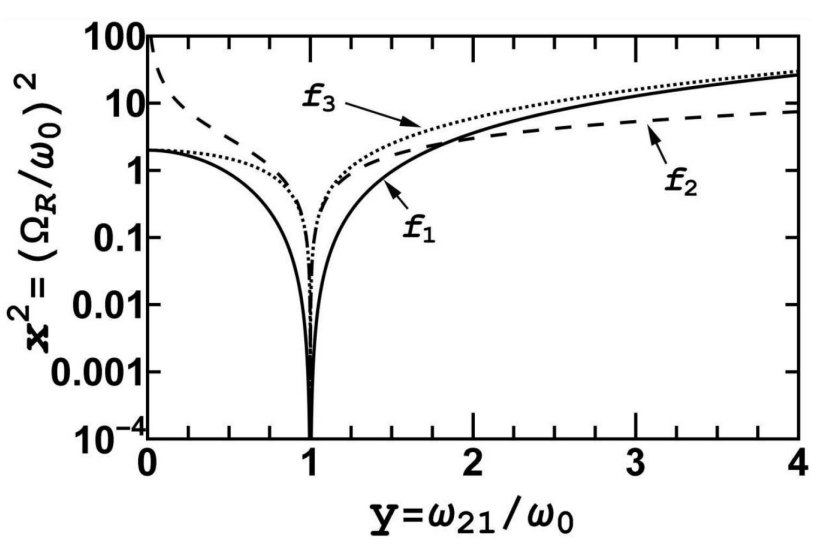

Fig. 1. The functions $f_{1}(y), f_{2}(y)$ and $f_{3}(y)$ given by Eqs. (13), (30), and (31), respectively, in their dependence on the laser frequency parameter $y=\omega_{21} / \omega_{0}$, where $\omega_{21}$ is the transition frequency and $\omega_{0}$ - the laser frequency.

$$
2 \int_{t^{\prime}}^{t} \dot{R}_{0}^{*}\left(t^{\prime \prime}\right) R_{0}\left(t^{\prime \prime}\right) \mathrm{d} t^{\prime \prime}=\mathrm{i}\left(U\left(t^{\prime}\right)-U(t)\right),
$$

where

$$
\begin{aligned}
& U\left(t^{\prime}\right)=a \int f^{2}\left(t^{\prime}\right) \mathrm{d} t^{\prime}+b f^{2}\left(t^{\prime}\right) \sin \left(2 \omega_{0} t^{\prime}\right) \\
& \quad+\mathrm{i} c f^{2}\left(t^{\prime}\right) \cos \left(2 \omega_{0} t^{\prime}\right)
\end{aligned}
$$

with

$$
a=x^{2} \frac{y}{y^{2}-1} \omega_{0}, \quad b=\frac{a}{2 \omega_{0}}, \quad c=\frac{b}{y}=\frac{a}{2 \omega_{21}} .
$$

We recognize in $a$ the standard Stark shift of the transition frequency, while $b(c)$ is the ratio of this shift to double laser (transition) frequency. Due to (14), we have $Z\left(t, t^{\prime}\right)=\exp \left(\mathrm{i}\left(U\left(t^{\prime}\right)-U(t)\right)\right)$ and

$$
\begin{aligned}
& \frac{\mathrm{d} Z\left(t, t^{\prime}\right)}{\mathrm{d} t^{\prime}}= \\
& \mathrm{i} a f^{2}\left(t^{\prime}\right)\left(1+\cos \left(2 \omega_{0} t^{\prime}\right)-\frac{\mathrm{i}}{y} \sin \left(2 \omega_{0} t^{\prime}\right)\right) Z\left(t, t^{\prime}\right)
\end{aligned}
$$

after neglecting the minor contribution coming from $\mathrm{d}\left(f^{2}\left(t^{\prime}\right)\right) / \mathrm{d} t^{\prime}$. As a result, the integrand in (10) is

$$
\begin{aligned}
R_{0}\left(t^{\prime}\right) \frac{\mathrm{d} Z\left(t, t^{\prime}\right)}{\mathrm{d} t^{\prime}}=\frac{i}{2} \frac{x^{3} y \omega_{0}}{\left(y^{2}-1\right)^{2}} f^{3}\left(t^{\prime}\right) \mathrm{e}^{\mathrm{i} \omega_{21} t^{\prime}} Z\left(t, t^{\prime}\right) \\
\times\left[\left(\frac{3}{2} y-\frac{1}{2 y}-1\right) \mathrm{e}^{\mathrm{i} \omega_{0} t^{\prime}}+\left(\frac{3}{2} y-\frac{1}{2 y}+1\right) \mathrm{e}^{-\mathrm{i} \omega_{0} t^{\prime}}\right. \\
\left.+\left(\frac{1}{2} y+\frac{1}{2 y}-1\right) \mathrm{e}^{\mathrm{i} 3 \omega_{0} t^{\prime}}+\left(\frac{1}{2} y+\frac{1}{2 y}+1\right) \mathrm{e}^{-\mathrm{i} 3 \omega_{0} t^{\prime}}\right] .
\end{aligned}
$$

Then, we apply to $Z\left(t, t^{\prime}\right)$ the Fourier-Bessel expansions $[24,25]$ :

$$
\mathrm{e}^{\mathrm{i} q \sin (\varphi)}=\sum_{n=-\infty}^{\infty} J_{n}(q) \mathrm{e}^{\mathrm{i} n \varphi},
$$

$$
\mathrm{e}^{q \cos (\varphi)}=\sum_{n=-\infty}^{\infty} I_{n}(q) \mathrm{e}^{\mathrm{i} n \varphi},
$$

where $n$ runs all positive and negative integers, $J_{n}(q)$ is the first kind Bessel function, and $I_{n}(q)$ is the modified Bessel function. For positive $n$, the Bessel function is represented by the series

$$
J_{n}(q)=\sum_{k=0}^{\infty}(-1)^{k} \frac{\left(\frac{q}{2}\right)^{n+2 k}}{k !(n+k) !},
$$

while the series for $I_{n}(q)$ is obtained from the above one by removing the factor $(-1)^{n}$. For negative $n$, one needs to use the relations $J_{-n}(q)=(-1)^{n} J_{n}(q)$ and $I_{-n}(q)=I_{n}(q)$. After applying these expansions to $Z\left(t, t^{\prime}\right)$, we shift appropriately the summation indices in (18) to obtain the common function $\exp \left(\mathrm{i}\left(\omega_{21}+(2 n+2 m+1) \omega_{0}\right) t^{\prime}+a \int f^{2}\left(t^{\prime}\right) \mathrm{d} t^{\prime}\right)$ for all terms. Then, we assume the lack of any higherorder odd-photon resonance in the system $\left(\omega_{21} \neq N \omega_{0}\right.$ with $N$ being positive odd number) and such detuning from this resonance that the Stark shift $|a| \ll\left|\left(\omega_{21}+(2 n+2 m+1) \omega_{0}\right)\right|$. As a consequence, we can integrate Eq. (18) over $t^{\prime}$, in order to obtain $R_{1}$ from Eq. (10), using the procedure described at the beginning of Sect. 3. As a result we obtain

$$
\begin{aligned}
& R_{1}(t)=-\frac{f^{3}(t)}{4} \frac{x^{3} y}{\left(y^{2}-1\right)^{2}} \\
& \times \sum_{n, m, n^{\prime}, m^{\prime}=-\infty}^{\infty} \frac{A_{n, m}^{n^{\prime}, m^{\prime}}}{y+2(n+m)+1} \\
& \quad \times \exp \left(\mathrm{i} \omega_{21} t+\mathrm{i}\left(2 n+2 m-2 n^{\prime}+2 m^{\prime}+1\right) \omega_{0} t\right),
\end{aligned}
$$

where

$$
\begin{aligned}
& A_{n, m}^{n^{\prime}, m^{\prime}}=(-1)^{m} J_{n^{\prime}}\left(b f^{2}(t)\right) I_{m}\left(c f^{2}(t)\right) I_{m^{\prime}}\left(c f^{2}(t)\right) \\
& \quad \times\left[\left(\frac{y}{2}+\frac{1}{2 y}-1\right) J_{n-1}\left(b f^{2}(t)\right)\right. \\
& +\left(\frac{3 y}{2}-\frac{1}{2 y}-1\right) J_{n}\left(b f^{2}(t)\right) \\
& +\left(\frac{3 y}{2}-\frac{1}{2 y}+1\right) J_{n+1}\left(b f^{2}(t)\right) \\
& \left.\quad+\left(\frac{y}{2}+\frac{1}{2 y}+1\right) J_{n+2}\left(b f^{2}(t)\right)\right]
\end{aligned}
$$

and $y+2(n+m)+1$ needs to be non-zero.

\section{Representative results}

\subsection{General formula for the dipole moment}

We use the approximate solution $R(t)=R_{0}(t)+R_{1}(t)$ to Eq. (1), with $R_{0}(t)$ given by $(12)$ and $R_{1}(t)$ by $(22)$, to write Eq. (7) for the induced electric dipole moment as $\boldsymbol{d}(t)=\frac{\boldsymbol{d}_{0}(t)+\boldsymbol{d}_{1}(t)}{1+|R|^{2}}$, where 


$$
\boldsymbol{d}_{0}(t)=2 \boldsymbol{\mu}_{12} \operatorname{Re}\left(R_{0}(t) \mathrm{e}^{-\mathrm{i} \omega_{21} t}\right)=
$$

$$
2 \boldsymbol{\mu}_{12} \frac{x y}{y^{2}-1} f(t) \cos \left(\omega_{0} t\right)
$$

and

$$
\begin{aligned}
& \boldsymbol{d}_{1}(t)=2 \boldsymbol{\mu}_{12} \operatorname{Re}\left(R_{1}(t) \mathrm{e}^{-\mathrm{i} \omega_{21} t}\right)=-\frac{\boldsymbol{\mu}_{12} f^{3}(t)}{2} \frac{x^{3} y}{\left(y^{2}-1\right)^{2}} \\
& \times \sum_{n, m, n^{\prime}, m^{\prime}=-\infty}^{\infty} A_{n, m}^{n^{\prime}, m^{\prime}} \frac{\cos \left(\left(2\left(n+m-n^{\prime}+m^{\prime}\right)+1\right) \omega_{0} t\right)}{y+2(n+m)+1} .
\end{aligned}
$$

Since $|R|^{2} \ll 1$ for weak excitations, the dipole moment is (to the first approximation) determined by the sum of Eqs. (24) and (25) only. The part $\boldsymbol{d}_{0}(t)$, coming from $R_{0}$, is known from the standard first-order perturbation theory when this theory is applied to (2) and (3) for the population amplitudes $C_{1}$ and $C_{2}$. The other part, $\boldsymbol{d}_{1}(t)$, comes from our $R_{1}$ (Eq. (22)) obtained by solving analytically the Riccati-type Eq. (1) with the use of the linearization procedure. Part $\boldsymbol{d}_{1}(t)$ describes the generation of odd-order laser harmonics by the two-level system. The present $\boldsymbol{d}_{1}(t)$ covers the case of a smooth pulse, $f(t)$, of arbitrary frequency, $\omega_{0}$, and thus broadens substantially the earlier results, ([19], part 4.1) and ([5(b), part II), obtained along different lines for the square pulse of low frequency $(y \gg 1)$ only. We mention that the coefficients $A_{n, m}^{n^{\prime}, m^{\prime}}$ include the Bessel functions of arguments dependent on $x=\Omega_{R} / \omega_{0}$, where $\Omega_{R}=\boldsymbol{\mu}_{21} \cdot \varepsilon_{0} / \hbar$, thus each frequency component of $\boldsymbol{d}_{1}(t)$ depends on a series of different powers of both the electric field $\varepsilon_{0}$ and the transition dipole $\boldsymbol{\mu}_{21}$.

\subsection{Particular limits of $\boldsymbol{d}_{1}(t)$}

In different (physically essential) limits (25) takes much simpler forms.

\subsubsection{Case $y \gg 1$}

First, we consider the case of low laser frequencies $\left(\omega_{0} \ll \omega_{21}, y \gg 1\right)$. In this case, the parameter $b$ from (16) converts into $b^{\prime}=\frac{y}{2}(x / y)^{2}$, while $c$ into $c^{\prime}=\frac{1}{2}(x / y)^{2}$. For $y \gg 1$, we however have the restriction $(x / y)^{2} \ll 2$ resulting from the zero-order solution $R_{0}$ (see (12) and (13)). Thus, we always have $c^{\prime} \ll 1$. It means that the two generalized Bessel functions $I_{\nu}$ in (23) are very close to 1 for $m=m^{\prime}=0$ and are very close to zero for other indices $m$ and $m^{\prime}$. We are, thus, allowed to make the approximations $I_{m}\left(c^{\prime} f^{2}(t)\right)=\delta_{m, 0}$ and $I_{m^{\prime}}\left(c^{\prime} f^{2}(t)\right)=\delta_{m^{\prime}, 0}$, where $\delta_{\alpha, \beta}$ is the Kronecker symbol. For $y \gg 1$, it is also justified to retain only the leading term, linear in $y$, in the coefficients at the Bessel functions $J_{\nu}$ in (23). In the limit of $y \gg 1$, Eq. (25) for $\boldsymbol{d}_{1}(t)$ is thus reduced to

$$
\begin{aligned}
& \boldsymbol{d}_{1}(t) \stackrel{y \gg 1}{=}-\frac{\boldsymbol{\mu}_{12}}{4}\left(\frac{x}{y}\right)^{3} f^{3}(t) \\
& \times \sum_{n, n^{\prime}=-\infty}^{\infty} B_{n}^{n^{\prime}} \frac{\cos \left(\left(2\left(n-n^{\prime}\right)+1\right) \omega_{0} t\right)}{1+\frac{2 n+1}{y}},
\end{aligned}
$$

where

$$
\begin{aligned}
& B_{n}^{n^{\prime}}=3\left[J_{n}\left(b^{\prime} f^{2}(t)\right)+J_{n+1}\left(b^{\prime} f^{2}(t)\right)\right] J_{n^{\prime}}\left(b^{\prime} f^{2}(t)\right) \\
& \quad+\left[J_{n-1}\left(b^{\prime} f^{2}(t)\right)+J_{n+2}\left(b^{\prime} f^{2}(t)\right)\right] J_{n^{\prime}}\left(b^{\prime} f^{2}(t)\right) .
\end{aligned}
$$

If $b^{\prime} \ll 1$, the coefficient $B_{n}^{n^{\prime}}$ can be further simplified by retaining only the leading term in the series representation of a given Bessel function $J_{\nu}\left(b^{\prime} f^{2}(t)\right)$. One can see that, for a reasonable $y \approx 10$, the parameter $b^{\prime}$ remains much smaller than 1 even for $x$ as large as 1, i.e., for nominally strong fields.

\subsubsection{Case $y \ll 1$}

In the opposite case of high laser frequencies $\left(\omega_{0} \gg \omega_{21}, y \ll 1\right)$, the parameters $b$ and $c$ convert into $b^{\prime \prime}=-x^{2} y / 2$ and $c^{\prime \prime}=b^{\prime \prime} / y=-x^{2} / 2$, respectively. Both $\left|b^{\prime \prime}\right|$ and $\left|c^{\prime \prime}\right|$ are much smaller than 1 because the applicability condition for the zero-order solution $R_{0}$ requires $x^{2} \ll 2$ when $y \ll 1$ (see (12) and (13)). Now, $1 / y$ becomes the dominant term in the coefficients at the Bessel functions $J_{\nu}$ in (23). With only this term retained, Eq. (25) for $\boldsymbol{d}_{1}(t)$ transforms, for $y \ll 1$, into the simpler form

$$
\begin{aligned}
& \boldsymbol{d}_{1}(t) \stackrel{y \ll 1}{=}-\frac{\boldsymbol{\mu}_{12}}{4} x^{3} f^{3}(t) \\
& \times \sum_{n, m, n^{\prime}, m^{\prime}=-\infty}^{\infty} C_{n, m}^{n^{\prime}, m^{\prime}} \frac{\cos \left(\left(2\left(n+m-n^{\prime}+m^{\prime}\right)+1\right) \omega_{0} t\right)}{y+2(n+m)+1}
\end{aligned}
$$

where

$$
\begin{aligned}
& C_{n, m}^{n^{\prime}, m^{\prime}}=(-1)^{m} J_{n^{\prime}}\left(b^{\prime \prime} f^{2}(t)\right) I_{m}\left(c^{\prime \prime} f^{2}(t)\right) I_{m^{\prime}}\left(c^{\prime \prime} f^{2}(t)\right) \\
& \quad \times\left[J_{n-1}\left(b^{\prime \prime} f^{2}(t)\right)-J_{n}\left(b^{\prime \prime} f^{2}(t)\right)\right. \\
& \left.\quad-J_{n+1}\left(b^{\prime \prime} f^{2}(t)\right)+J_{n+2}\left(b^{\prime \prime} f^{2}(t)\right)\right] .
\end{aligned}
$$

\subsubsection{Case $y=0$}

Equations (28) and (29) cover the limiting case of the degenerate two-level system $\left(\omega_{21}=0, y=0\right)$. In this case, $b^{\prime \prime}=0$, and $c^{\prime \prime}$ still keeps a small non-zero value $-x^{2} / 2$. Thus, each Bessel function $J_{\nu}$ in (29) behaves like the Kronecker symbol and the coefficient $C_{n, m}^{n^{\prime}, m^{\prime}}$ is made proportional to $\left(\delta_{n, 1}-\delta_{n, 0}-\delta_{n,-1}+\right.$ $\left.\delta_{n,-2}\right) \delta_{n^{\prime}, 0}$. When performing the summation over $n$ and $n^{\prime}$, as required by (28), we find that the contributions from $\delta_{n, 1}$ and $\delta_{n,-2}$ cancel mutually, since $I_{\nu}=I_{-\nu}$, and the same concerns the contributions from $\delta_{n, 0}$ and $\delta_{n,-1}$. As a result, $\boldsymbol{d}_{1}(t)$ from (28) becomes zero for $y=0$. Consequently, no dipole moment is induced in the degenerate two-level system since $\boldsymbol{d}_{0}(t)$ given by Eq. (24) is also zero when $y=0$. This conclusion is consistent with the general outcome of the exact Riccati Eq. (1) for $\omega_{21}=0$. In the limit $\omega_{21}=0$, the coupling parameter $Q(t)$ given by (4) is made real and Eq. (1) has the exact solution 


$$
R(t)=\mathrm{i} \tan \left(\int_{t_{0}}^{t} Q\left(t^{\prime}\right) \mathrm{d} t^{\prime}\right)
$$

for any $Q(t)$. As purely imaginary for $\omega_{21}=0$, this solution produces no induced dipole moment from (7).

\subsubsection{Arbitrary y but both $|b| \ll 1$ and $|c| \ll 1$}

Now, we focus on the case of arbitrary laser frequencies $(y)$ but such field strengths $(x)$ that both $|b| \ll 1$ and $|c| \ll 1$. Then, each Bessel function $J_{\nu}$ and each modified Bessel function $I_{\nu}$ in (23) for $A_{n, m}^{n^{\prime}, m^{\prime}}$ can be approximated by the first term in its series representation (see (21)). Moreover, we are also allowed to retain in (23) only what results from the Bessel function $J_{\nu}$ of the lowest order $|\nu|$. Due to (16), these approximations should work well when the parameters $x$ and $y$ fulfil the set of two inequalities

$$
\begin{aligned}
& x^{2} \ll f_{2}(t)=2 \frac{\left|y^{2}-1\right|}{y}, \\
& x^{2} \ll f_{3}(t)=2\left|y^{2}-1\right| .
\end{aligned}
$$

In Fig. 1, we present the functions $f_{2}$ (dash line) and $f_{3}$ (dot line) versus $y$ and compare them with the function $f_{1}$ (solid line) defined by (13) and imposing additional restriction on $x^{2}$. These three restrictions, namely Eqs. (13), (30), and (31), have to be reconciled simultaneously. Thus, for a given $y$, the strength parameter $x$ needs to be chosen as that satisfying the inequality $x^{2} \ll \min \left(f_{1}(y), f_{2}(y), f_{3}(y)\right)$, where $\min \left(f_{1}, f_{2}, f_{3}\right)$ means the smallest function of the three ones. Figure 1 can be helpful in quick estimation of the related parameters $x$ and $y$ for which one can apply the above described approximations.

With the coefficients $A_{n, m}^{n^{\prime}, m^{\prime}}$ approximated in this way, we are able to find in compact forms the components of $\boldsymbol{d}_{1}(t)$ oscillating at frequencies $\omega_{0}, 3 \omega_{0}, 5 \omega_{0}, \ldots$ We denote a given frequency by $N \omega_{0}$, where $N=\left|2\left(n+m-n^{\prime}+m^{\prime}\right)+1\right|$, and a given component by $\boldsymbol{d}_{1}^{N \omega_{0}}(t)$. Thus, Eq. (25) is replaced by $\boldsymbol{d}_{1}(t)=$ $\sum_{N} \boldsymbol{d}_{1}^{N \omega_{0}}(t)$. Here, we are not interested in giving $\boldsymbol{d}_{1}^{1 \omega_{0}}(t)$ because it is a minor, nonlinear in $x$ addition to $\boldsymbol{d}_{0}(t)$ defined by $(24)$. As examples, we give instead the formulae for $\boldsymbol{d}_{1}^{3 \omega_{0}}(t)$ and $\boldsymbol{d}_{1}^{5 \omega_{0}}(t)$. According to (25), infinite number of combinations $\left(n, m, n^{\prime}, m^{\prime}\right)$ constitute a fixed $N$, in general. However, the assumption that both $|b| \ll 1$ and $|c| \ll 1$ means, fortunately, that among these combinations there are ones bringing dominant contribution to the coefficients $A_{n, m}^{n^{\prime}, m^{\prime}}$. This dominant contribution comes from as small as possible indices $n, m, n^{\prime}, m^{\prime}$ leading to the fixed $N$. For $N=3$, the dominant contribution was found to come from the combinations $(1,0,0,0)$ and $(-2,0,0,0)$. As a result, the leading part in both $A_{1,0}^{0,0}$ and $A_{-2,0}^{0,0}$ is proportional to $J_{0}^{2}\left(b f^{2}(t)\right) I_{0}^{2}\left(c f^{2}(t)\right) \approx 1$. However, the number of essential combinations increases rapidly with increasing $N$. For $N=5$, one finds eight such combinations, namely $(2,0,0,0),(1,0,-1,0),(1,1,0,0),(1,0,0,1),(-3,0,0,0)$,
$(-2,0,1,0),(-2,-1,0,0)$, and $(-2,0,0,-1)$. Any above combination generates the leading part in the relevant coefficient $A_{n, m}^{n^{9}, m^{\prime}}$ proportional to either $b f^{2}(t) \sim x^{2}$, or $c f^{2}(t) \sim x^{2}$. The leading parts in the coefficients $A_{n, m}^{n^{\prime}, m^{\prime}}$, found along this line, give the following close-form components $\boldsymbol{d}_{1}^{3 \omega_{0}}(t)$ and $\boldsymbol{d}_{1}^{5 \omega_{0}}(t)$ of $\boldsymbol{d}_{1}(t)$ :

$$
\begin{aligned}
& \boldsymbol{d}_{1}^{3 \omega_{0}}(t)=-\frac{\boldsymbol{\mu}_{12}}{2} \frac{x^{3} y}{\left(y^{2}-1\right)^{2}} \frac{y^{2}+7}{y^{2}-9} f^{3}(t) \cos \left(3 \omega_{0} t\right), \\
& \boldsymbol{d}_{1}^{5 \omega_{0}}(t)=\frac{\boldsymbol{\mu}_{12}}{4} \frac{x^{5} y}{\left(y^{2}-1\right)^{3}} \frac{y^{4}+42 y^{2}+53}{\left(y^{2}-9\right)\left(y^{2}-25\right)} \\
& \times f^{5}(t) \cos \left(5 \omega_{0} t\right) .
\end{aligned}
$$

Obviously, the above formulae are valid outside the exact odd-order resonances, i.e., for $y \neq 1,3$ in (32) and $y \neq 1,3,5$ in (33). As seen, the $N$-th component oscillating at frequency $N \omega_{0}$ is made proportional to $x^{N}$ in the approximation of both $|b| \ll 1$ and $|c| \ll 1$. With increase in $N$, the dependence of the $N$-th component on the laser frequency parameter $y=\omega_{21} / \omega_{0}$ gets more and more complicated due to increase in the number of essential combinations $\left(n, m, n^{\prime}, m^{\prime}\right)$ that have to be included.

\subsection{Dipole moment when both $|b| \ll 1$ and $|c| \ll 1$}

Now, we estimate the effect of the denominator in the definition of the dipole moment, $\boldsymbol{d}(t)=\frac{\boldsymbol{d}_{0}(t)+\boldsymbol{d}_{1}(t)}{1+|R|^{2}}$, on $\boldsymbol{d}(t)$. Since $|R|^{2} \ll 1$ for weak excitations, this effect can be found by replacing $|R|$ by $\left|R_{0}\right| \gg\left|R_{1}\right|$ and using the power expansion

$$
\left(1+\left|R_{0}\right|^{2}\right)^{-1} \approx 1-\left|R_{0}\right|^{2}+\left(\left|R_{0}\right|^{2}\right)^{2}-\left(\left|R_{0}\right|^{2}\right)^{3}+\ldots
$$

Along this line we get $\boldsymbol{d}(t)=\boldsymbol{d}_{0}(t)+\boldsymbol{d}_{1}(t)+\boldsymbol{d}_{\text {cor }}(t)$, where the correcting term is

$$
\begin{aligned}
& \boldsymbol{d}_{\mathrm{cor}}(t)=-\left(\boldsymbol{d}_{0}(t)+\boldsymbol{d}_{1}(t)\right) \\
& \quad \times\left(\left|R_{0}\right|^{2}-\left(\left|R_{0}\right|^{2}\right)^{2}+\left(\left|R_{0}\right|^{2}\right)^{3}-\ldots\right) .
\end{aligned}
$$

As an example, we shall find this correction under the same assumptions as in Sect. 4.2.4, i.e., that both $|b| \ll 1$ and $|c| \ll 1$.

In analogy to the previous decomposition $\boldsymbol{d}_{1}(t)=\sum_{N} \boldsymbol{d}_{1}^{N \omega_{0}}(t)$, we write $\boldsymbol{d}_{\mathrm{cor}}(t)=\sum_{N} \boldsymbol{d}_{\mathrm{cor}}^{N \omega_{0}}(t)$, where the component $\boldsymbol{d}_{\mathrm{cor}}^{N \omega_{0}}(t)$ of the total correction oscillates at frequency $N \omega_{0}$. A given component $\boldsymbol{d}_{\text {cor }}^{N \omega_{0}}(t)$ can be obtained elementarily by using (12), (24), (32) and (33). The component $\boldsymbol{d}_{\mathrm{cor}}^{1 \omega_{0}}(t)$ was found to be nonlinear in $x$ and, as much smaller than $\boldsymbol{d}_{0}(t)$, it was rejected. However, the components $\boldsymbol{d}_{\mathrm{cor}}^{N \omega_{0}}(t)$, for $N \geq 3$, turned out to be comparable to $\boldsymbol{d}_{1}^{N \omega_{0}}(t)$ given by $(32)$ and (33). We have found that, for $N=3$, the leading contribution $\left(\sim x^{3}\right)$ to $\boldsymbol{d}_{\mathrm{cor}}^{3 \omega_{0}}(t)$ comes from $\boldsymbol{d}_{0}(t)\left|R_{0}\right|^{2}$ and is

$$
\boldsymbol{d}_{\mathrm{cor}}^{3 \omega_{0}}(t)=-\frac{\boldsymbol{\mu}_{12}}{2} \frac{x^{3} y}{\left(y^{2}-1\right)^{2}} f^{3}(t) \cos \left(3 \omega_{0} t\right) .
$$




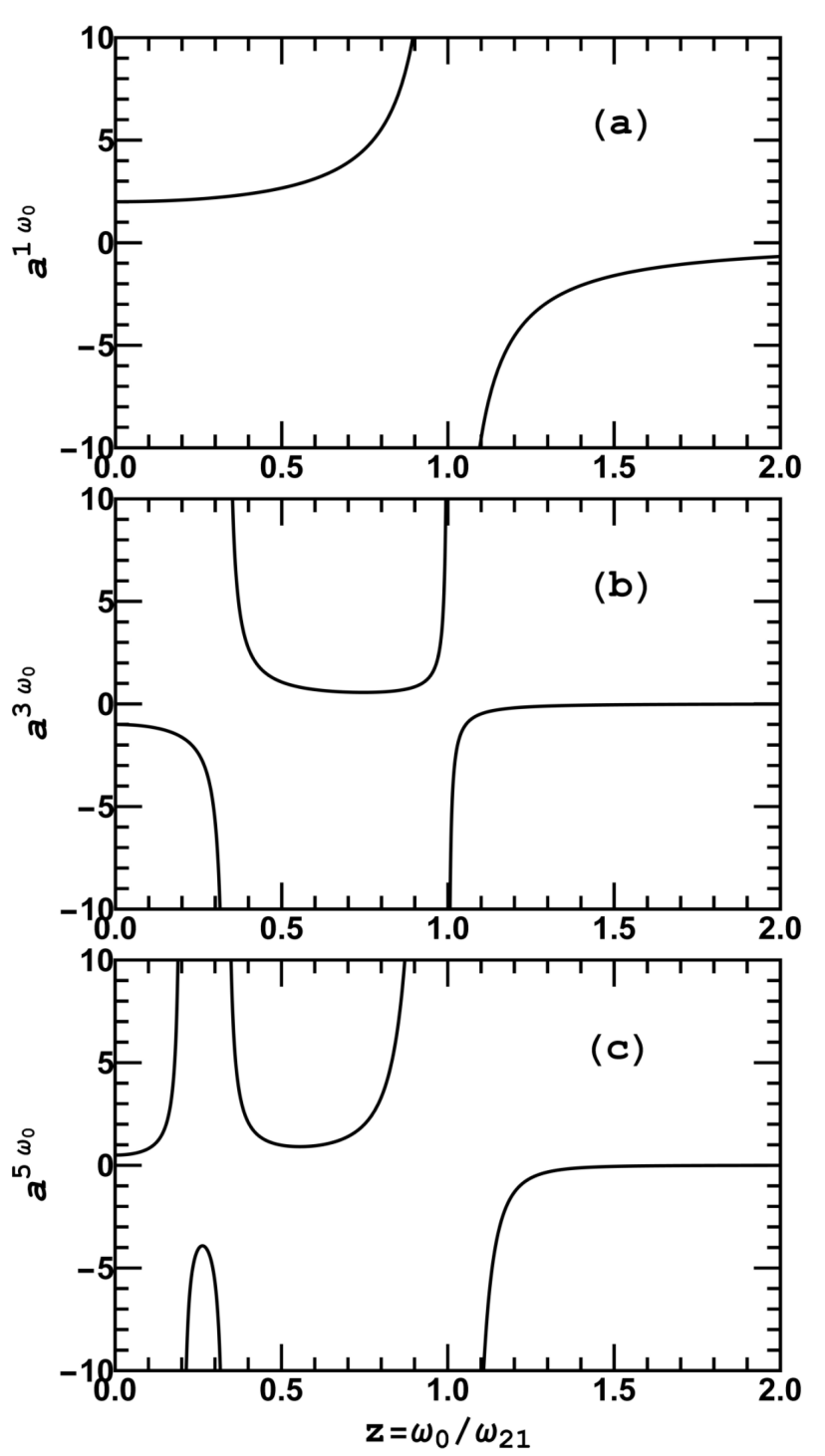

Fig. 2. The coefficients $a^{N \omega_{0}}(z)=\frac{d^{N \omega_{0}(t)}}{\mu_{12}} \rho^{N} f^{N}(t)$ $\times \cos \left(N \omega_{0} t\right)$ in Eqs. (37)-(39) versus the laser frequency parameter $z=\omega_{0} / \omega_{21}$, for $N=1,3$ and 5 . The vertical asymptotes are at $z=1$ in (a), $z=1 / 3$ and 1 in (b), $z=1 / 5,1 / 3$ and 1 in (c).

For $N=5$, the leading contribution $\left(\sim x^{5}\right)$ to $\boldsymbol{d}_{\mathrm{cor}}^{5 \omega_{0}}(t)$ takes its origin in both $\boldsymbol{d}_{0}(t)\left(\left|R_{0}\right|^{2}\right)^{2}$ and $\boldsymbol{d}_{1}^{3 \omega_{0}}(t)\left|R_{0}\right|^{2}$ and amounts to

$$
\boldsymbol{d}_{\mathrm{cor}}^{5 \omega_{0}}(t)=\frac{\boldsymbol{\mu}_{12}}{4} \frac{x^{5} y}{\left(y^{2}-1\right)^{2}\left(y^{2}-9\right)} f^{5}(t) \cos \left(5 \omega_{0} t\right) .
$$

After including those corrections, the dipole moment is $\boldsymbol{d}(t)=\boldsymbol{d}_{0}(t)+\boldsymbol{d}_{1}(t)+\boldsymbol{d}_{\mathrm{cor}}(t)=\sum_{N} \boldsymbol{d}^{N \omega_{0}}(t)$, where $\boldsymbol{d}^{N \omega_{0}}(t)$ means the leading term in the dipole component oscillating at frequency $N \omega_{0}$. Obviously, $\boldsymbol{d}^{1 \omega_{0}}(t)=\boldsymbol{d}_{0}(t)$ and is given by Eq. (24). For $N \geq 3$, we however have $\boldsymbol{d}^{N \omega_{0}}(t)=\boldsymbol{d}_{1}^{N \omega_{0}}(t)+\boldsymbol{d}_{\text {cor }}^{N \omega_{0}}(t)$. In conformity with Eqs. (24), (32), (33), (35), and (36), one obtains

$$
\begin{aligned}
& \boldsymbol{d}^{1 \omega_{0}}(t)=\boldsymbol{d}_{0}(t)=2 \boldsymbol{\mu}_{12} \frac{x y}{y^{2}-1} f(t) \cos \left(\omega_{0} t\right), \\
& \boldsymbol{d}^{3 \omega_{0}}(t)=-\boldsymbol{\mu}_{12} \frac{x^{3} y}{\left(y^{2}-1\right)\left(y^{2}-9\right)} f^{3}(t) \cos \left(3 \omega_{0} t\right), \\
& \boldsymbol{d}^{5 \omega_{0}}(t)=\boldsymbol{\mu}_{12} \frac{\left(x^{5} y\right)\left(y^{4}+8 y^{2}+39\right)}{\left(y^{2}-1\right)^{3}\left(y^{2}-9\right)\left(y^{2}-25\right)} \\
& \quad \times \frac{f^{5}(t) \cos \left(5 \omega_{0} t\right)}{2} .
\end{aligned}
$$

Since $x=\Omega_{R} / \omega_{0}$, where $\Omega_{R}=\mu_{21} \cdot \varepsilon_{0} / \hbar$, the leading term in a given dipole component is proportional to the appropriate even power of the transition dipole $\boldsymbol{\mu}_{21}$ and the appropriate odd power of the electric field amplitude $\varepsilon_{0}$.

To draw the time-independent factors in (37)-(39) as functions of laser frequency $\omega_{0}$, we introduce the parameters $\rho=\Omega_{R} / \omega_{21}$ and $z=y^{-1}=\omega_{0} / \omega_{21}$ linked to $x$ through the relation $\rho / z=x$. As distinct from $x$ and $y$, the present laser strength and laser frequency parameters $\rho$ and $z$, respectively, have the transition frequency $\omega_{21}$ in their denominators. After expressing (37)-(39) in the language of $\rho$ and we go to the coefficients $a^{N \omega_{0}}(z)=\boldsymbol{d}^{N \omega_{0}}(t) / \boldsymbol{\mu}_{12} \rho^{N} f^{N}(t) \cos \left(N \omega_{0} t\right)$ completely determining the dependence of the amplitudes of the dipole components $\boldsymbol{d}^{N \omega_{0}}(t)$ on laser frequency parameter $z=\omega_{0} / \omega_{21}$. This dependence is shown in Fig. 2 .

\subsection{Field-dependent correction to $\boldsymbol{d}^{1 \omega_{0}}(t)$}

Next, we calculate the nonlinear in $x$ correction to the dipole component $\boldsymbol{d}^{1 \omega_{0}}(t)=\boldsymbol{d}_{0}(t)$ under the assumption that both $|b| \ll 1$ and $|c| \ll 1$. According to Sect. 4.2.4 and Eq. (34), this correction is defined as $\boldsymbol{d}_{1}^{1 \omega_{0}}(t)-$ $\left(\boldsymbol{d}_{0}(t)\left|R_{0}\right|^{2}\right)^{\omega_{0}}-\left(\boldsymbol{d}_{1}^{1 \omega_{0}}(t)\left|R_{0}\right|^{2}\right)^{\omega_{0}}$, where $A^{\omega_{0}}$ means the part of $A$ oscillating at frequency $\omega_{0}$. To find $\boldsymbol{d}_{1}^{1 \omega_{0}}(t)$ we put $2\left(n+m-n^{\prime}+m^{\prime}\right)+1= \pm 1$ in Eq. (25) for $\boldsymbol{d}_{1}(t)$ and take into account only the combinations $\left(n, m, n^{\prime}, m^{\prime}\right)$ of as small as possible values of the indices, i.e., $(0,0,0,0)$ and $(-1,0,0,0)$. Then, Eq. (25) gives

$$
\boldsymbol{d}_{1}^{1 \omega_{0}}(t)=-\frac{\boldsymbol{\mu}_{12}}{2} \frac{x^{3} y}{\left(y^{2}-1\right)^{2}} F(y, t) f^{3}(t) \cos \left(\omega_{0} t\right)
$$

where

$$
\begin{aligned}
& F(y, t)=J_{0}\left(b f^{2}(t)\right) I_{0}^{2}\left(c f^{2}(t)\right) \\
& \quad \times\left[J_{2}\left(b f^{2}(t)\right)+\frac{2}{y} \frac{y^{2}+1}{y^{2}-1} J_{1}\left(b f^{2}(t)\right)\right. \\
& \left.\quad+\frac{3 y^{2}+1}{y^{2}-1} J_{0}\left(b f^{2}(t)\right)\right] .
\end{aligned}
$$

Equations (40), (37), (34) and (12) lead to

$$
\begin{aligned}
& \left(\boldsymbol{d}_{0}(t)\left|R_{0}\right|^{2}\right)^{\omega_{0}}=\frac{\boldsymbol{\mu}_{12}}{2} \frac{x^{3} y\left(3 y^{2}+1\right)}{\left(y^{2}-1\right)^{3}} f^{3}(t) \cos \left(\omega_{0} t\right), \\
& \left(\boldsymbol{d}_{1}^{1 \omega_{0}}(t)\left|R_{0}\right|^{2}\right)^{\omega_{0}}=-\frac{\boldsymbol{\mu}_{12}}{8} \frac{x^{5} y\left(3 y^{2}+1\right)}{\left(y^{2}-1\right)^{4}} \\
& \times F(y, t) f^{5}(t) \cos \left(\omega_{0} t\right) .
\end{aligned}
$$


Finally, we apply the series representations (Eq. (21)) for the Bessel functions $J_{\nu}$ and the modified Bessel functions $I_{\nu}$, inherent in $F(y, t)$, and group the terms with the same powers of $x$. With the correction calculated in this way, Eq. (37) is replaced by

$\boldsymbol{d}^{1 \omega_{0}}(t)=\boldsymbol{\mu}_{12} \sum_{n=0,2,4, \ldots} a_{n}(y)(x f(t))^{n+1} \cos \left(\omega_{0} t\right)$,

where, e.g.

$$
\begin{aligned}
& a_{0}(y)=\frac{2 y}{y^{2}-1}, \\
& a_{2}(y)=-y \frac{3 y^{2}+1}{\left(y^{2}-1\right)^{3}}, \\
& a_{4}(y)=\frac{y}{8} \frac{7 y^{4}+6 y^{2}+3}{\left(y^{2}-1\right)^{5}} .
\end{aligned}
$$

The first term in Eq. (44) is obviously equal to Eq. (37), while the other terms point to the dependence of the amplitude of the oscillation at frequency $\omega_{0}$ on higher powers of the laser strength parameter $x$. For a given $\omega_{0}$, these additional terms describe the dependence of the refractive index $n$ of the two-level system on strength of the laser field since from (44) and the Lorentz-Lorenz formula we get

$$
\begin{aligned}
n^{2} & =1+4 \pi N\left|\mu_{21}\right|^{2} \hbar \omega_{21} \\
& \times\left(b_{0}(z)+b_{2}(z)(\rho f(t))^{2}+b_{4}(z)(\rho f(t))^{4}+\ldots\right),
\end{aligned}
$$

where $N$ is the density of medium, $b_{n}(z)=a_{n}(y) / z^{n+1}$ with $z=\omega_{0} / \omega_{21}$ and $y=z^{-1}$, and $\rho=\Omega_{R} / \omega_{21}$. In Fig. 3, we show the dependence of the coefficients $b_{n}$ on the laser frequency parameter $z$.

To estimate the effect of laser strength on the refractive index we take as the two-level model the two lowest states in the hydrogen atom, i.e. the $1 S=|\rangle$ and $2 P=|\rangle$ states separated by the transition energy $\hbar \omega_{21}=10.2 \mathrm{eV}$. The electric field interacting with this system is assumed to come from the neodymium glass laser $\left(\hbar \omega_{0}=1.17 \mathrm{eV}\right)$. Consequently, the frequency parameter $y=\omega_{21} / \omega_{0}=8.72$ and corresponds to the regime of multiphoton excitation. Also, we take $x=0.3$ for the light strength parameter. Since $\left|\boldsymbol{r}_{21}\right|=z_{21}=4 \sqrt{2}(2 / 3)^{5}$ a.u., the taken $x$ means the laser electric field amplitude $\varepsilon_{0} \approx 0.017$ a.u. corresponding to the laser intensity $10^{13} \mathrm{~W} / \mathrm{cm}^{2}$. This is the highest available intensity because above it the model becomes questionable due to the neglect of the possible upper-state ionization. For the assumed values of $x$ and $y$, we find that both $|b|$ and $|c|$ are much smaller than 1 (precisely $b=5 \times 10^{-3}$ and $c=6 \times 10^{-4}$ from (16)) as required for applicability of Eq. (48). Since $z=1 / y=0.115$ and $\rho=x / y=0.0344$, the leading effect of laser strength on the refractive index comes from the term $b_{2}(z) \rho^{2}$ in (48). The ratio of this term to the standard strength-independent term $b_{0}(z)$ is evaluated as $b_{2}(z) \rho^{2} / b_{0}(z) \simeq-\frac{3}{2} \rho^{2}=-1.8 \times 10^{-3}$ for the taken frequency and strength of the laser field.

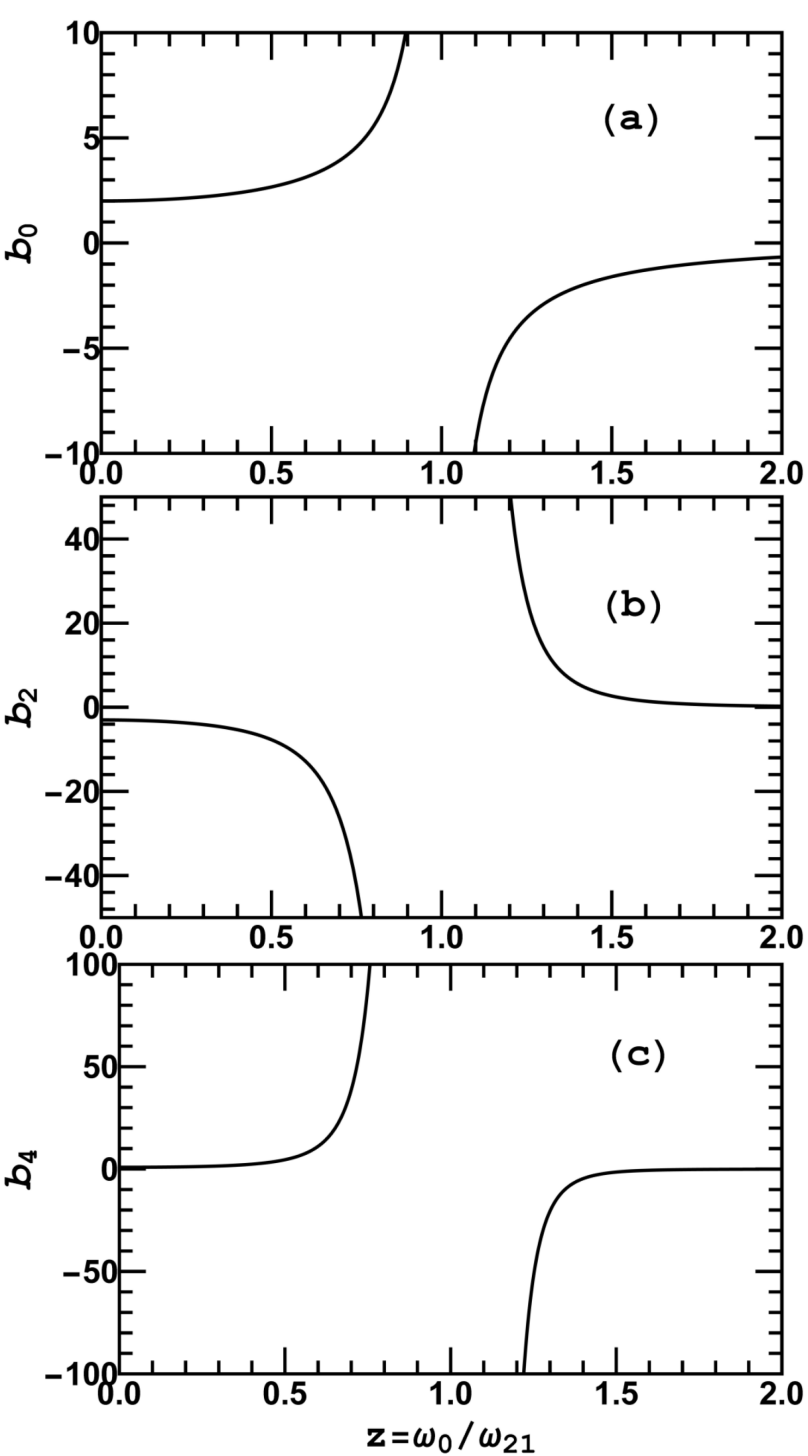

Fig. 3. The dependence of the coefficients $b_{n}(z)=$ $a_{n}(y=1 / z) / z^{n+1}$, given by Eqs. (45)-(47), on the laser frequency parameter $z=\omega_{0} / \omega_{21}$, for $n=0,2$ and 4 .

\section{Summary}

In this paper, we have worked with the appropriately linearized version of the exact, quadratically nonlinear Riccati-type differential equation for the ratio of the population amplitudes in a weakly laser-excited two-level system. First, we have analytically solved this linearized equation for arbitrary off-resonant laser frequencies and arbitrary smooth shapes of the laser pulse. Then, this solution has been used to derive an explicit formula for the laser-induced electric dipole moment in the system. Though complicated at the first sight, the formula has been shown to take substantially simpler forms in different laser frequency and laser strength limits. Also, we have used this formula to find some representative Fourier components of the induced dipole moment and 
discuss the dependence of the amplitudes of these components on the laser frequency and strength. To conclude, this paper shows that the linearized Riccati equation for population amplitudes is an effective analytical method for nonlinear optics of two-level systems weakly excited by a smooth laser pulse of frequency producing neither one-photon nor odd multiphoton resonances.

The assumption of a smooth laser pulse physically means that, in the time evolution of the pulse electric field $f(t) \cos \left(\omega_{0} t\right)$, the full width at half maximum (FWHM) of the pulse shape function $f(t)$ is much greater than the optical period $T=2 \pi / \omega_{0}$. It is well justified for all standard laser pulses except the recently celebrated few-cycle pulses. Under this reasonable assumption the time integral in Eq. (8) needs to be performed from the product of the slowly time varying shape function $f(t)$ and the function $\cos \left(\omega_{0} t\right) \exp \left(i \omega_{21} t\right)$ which is fast varying as long as $\omega_{0}$ substantially differs from $\omega_{21}$, i.e. in the absence of one-photon resonance. The absence of one-photon resonance practically means that the one-photon detuning $\left|\omega_{21}-\omega_{0}\right|$ well exceeds the spectral width of $f(t)$. In the present paper, we in fact were focused on this off-resonance case. The motivation for us was that in the majority of the ground-state atoms (except some alkali atoms), molecules, and ions the transition frequency to the first excited state, $\omega_{21}$, is higher or even much higher than a typical optical frequency $\omega_{0}$ (see Sect. 4.4, for example) and multiphoton excitation is rather met. In this case of our interest, the integration in Eq. (8) was performed by parts and only the dominant term $f(t)=\int_{t_{0}}^{t} \cos \left(\omega_{0} t^{\prime}\right) \exp \left(\mathrm{i} \omega_{21} t^{\prime}\right) \mathrm{d} t^{\prime}$ was retained. The above assumptions of smooth pulse and absence of one-photon resonance have led us to the explicit $R_{0}(t)$, given by (12), valid for an arbitrary pulse shape function $f(t)$. Thanks to this $R_{0}(t)$ we were then able to find general (22) for the small correction $R_{1}(t)$ applicable when no higher-order odd-photon resonance takes place in the two-level system.

The case of any odd-photon resonance in the system is more cumbersome. If one-photon resonance $\left(\omega_{0}=\omega_{21}\right)$ is present, the starting Eq. (8) for $R_{0}(t)$ splis into two integrals, namely $\frac{1}{2} \int_{t_{0}}^{t} f\left(t^{\prime}\right) \exp \left(2 \mathrm{i} \omega_{21} t^{\prime}\right) \mathrm{d} t^{\prime}$ and $\frac{1}{2} \int_{t_{0}}^{t} f\left(t^{\prime}\right) \mathrm{d} t^{\prime}$. For the assumed smooth-shape pulse, the first integral can be approximated by the leading term, - i $f(t) \exp \left(2 \mathrm{i} \omega_{21} t\right) / 4 \omega_{21}$ coming from integration by parts (see the beginning of Sect. 3). However, the other integral makes it impossible to find $R_{0}(t)$ in an explicit form valid for an arbitrary $f(t)$. As a matter of fact, we are forced to choose a given shape for $f(t)$ at this stage. For some shapes (e.g., the familiar $\left.f(t)=\sin ^{2}\left(\frac{\pi t}{t_{p}}\right)\right)$ the integral $\int_{t_{0}}^{t} f\left(t^{\prime}\right) \mathrm{d} t^{\prime}$ is expressed in terms of elementary functions, while for other shapes (e.g., the Gaussian shape $\left.f(t)=\exp \left(-t^{2} / t_{p}^{2}\right)\right)$ in terms of non-elementary functions (error function). Only in the first case, the small correction $R_{1}(t)$ could in principle be found along the analytical line similar to that presented in Sect. 3.
Consequently, no general expression like Eq. (22) can be obtained for the correction $R_{1}(t)$ when one-photon resonance takes place. If a higher-order odd-photon resonance is present, instead of one-photon resonance, then $R_{0}(t)$ given by Eq. (12) is still valid. However, Eq. (18), being the integrand in Eq. (10) for $R_{1}(t)$, needs to be separated into its resonant and off-resonant parts with the use of the Fourier-Bessel expansions given by Eqs. (19) and (20). Then, the off-resonant part of Eq. (18) can be integrated over time in the same way as described before Eq. (22). On the other hand, the resonant part should be integrated on its own foot. Moreover, all processes damping the upper level in more realistic two-level system (e.g., spontaneous emission and ionization) should be taken into account in the case of any odd-photon resonance. Phenomenogically, this damping can be included to the model by adding the term $-\mathrm{i} \frac{\gamma}{2} C_{2}$ to the right-hand side of Eq. (3), where $\gamma$ stands for the damping rate. As a consequence, the right-hand side of the Riccati Eq. (1) will be enriched by the extra term $-\mathrm{i} \frac{\gamma}{2} R$ leading to appropriate changes in Eq. (8) for $R_{0}$ and Eq. (9) for $R_{1}$. Thus, the above analysis shows that, at any exact and near odd-photon resonance, separate consideration of the two-level model would be necessary in the framework of linearized Riccati equation.

\section{References}

[1] L. Allen, J.H. Eberly, Optical Resonance and TwoLevel Atoms, Dover Publications, New York 1987.

[2] L. Plaja, L. Roso-Franco, J. Opt. Soc. Am. B 9, 2210 (1992); L. Plaja, L. Roso-Franco, J. Mod Opt. 40, 793 (1993).

[3] M.Y. Ivanov, P.B. Corkum, Phys. Rev. A 48, 580 (1993).

[4] M.Y. Ivanov, P.B. Corkum, P. Dietrich, Laser Phys. 3, 375 (1993).

[5] T. Zuo, S. Chelkowski, A.D. Bandrauk, Phys. Rev. A 48, 3837 (1993); T. Zuo, S. Chelkowski, A.D. Bandrauk, Phys. Rev. A 49, 3943 (1994).

[6] R. Bavli, Y. Dakhnovskii, Phys. Rev. A 48, R886 (1993).

[7] A. Levinson, M. Segev, G. Almogy, A. Yariv, Phys. Rev. A 49, R661 (1994).

[8] A.E. Kaplan, P.L. Shkolnikov, Phys. Rev. A 49, 1275 (1994).

[9] E. Fiordilino, V. Miceli, J. Mod Opt. 41, 1415 (1994); E. Fiordilino, V. Miceli, Laser Phys. 6, 1204 (1996)

[10] F.I. Gauthey, B.M. Garraway, P.L. Knight, Phys. Rev. A 56, 3093 (1997).

[11] P. Caldara, E. Fiordilino, J. Mod. Opt. 46, 743 (1999).

[12] S. Hughes, Phys. Rev. Lett. 81, 3363 (1998); S. Hughes, Phys. Rev. A 62, 055401 (2000).

[13] O.D. Mücke, T. Tritschler, M. Wegener, U. Morgner, F.X. Kärtner, Phys. Rev. Lett. 87, 057401 (2001).

[14] O.D. Mücke, T. Tritschler, M. Wegener, U. Morgner, F.X. Kärtner, Phys. Rev. Lett. 89, 127401 (2002). 
[15] C.F. de Morisson Faria, I. Rotter, Phys. Rev. A 66, 013402 (2002).

[16] T. Tritschler, O.D. Mücke, M. Wegener, U. Morgner, F.X. Kärtner, Phys. Rev. Lett. 90, 217404 (2003).

[17] T. Tritschler, O.D. Mücke, M. Wegener, Phys. Rev. A 68, 033404 (2003).

[18] D.F. Martinez, J. Phys. A Math. Gen. 38, 9979 (2005).

[19] A. Plucińska, R. Parzyński, J. Mod. Opt. 54, 745 (2007).

[20] M. Berent, R. Parzyński, Phys. Rev. A 80, 033834 (2009).

[21] A. Picón, L. Roso, J. Mompart, O. Varela, V. Ahufinger, R. Corbalán, L. Plaja, Phys. Rev. A 81, 033420 (2010).
[22] Y.V. Rostovtsev, H. Eleuch, A. Svidzinsky, H. Li, V. Sautenkov, M.O. Scully, Phys. Rev. A 79, 063833 (2009).

[23] M.O. Scully, M.S. Zubairy, Quantum Optics, Cambridge University Press, Cambridge 1997.

[24] I.S. Gradshteyn, I.M. Ryzhik, Table of Integrals, Series and Products, Parts 8.4-8.5, Academic Press, London 2000.

[25] M. Abramowitz, I.A. Stegun, Handbook of Mathematical Functions with Formulas, Graphs, and Mathematical Tables, Parts 9.1 and 9.6, Dover Publications, New York 1964. 